\title{
Cardiopulmonary exercise testing in systolic heart failure: from basic to advanced practice
}

\author{
Ugo Corrà
}

\author{
Cardiology Division, IRCCS Fondazione Salvatore Maugeri, Centro Medico e di Riabilitazione di Veruno, \\ Veruno (NO), Italy
}

\begin{abstract}
Cardiopulmonary exercise testing (CPET) is a specialized subtype of exercise testing that provides a more accurate and objective measure of cardiorespiratory fitness (CRF). CPET relies on measurement of ventilatory gases during exercise, i.e., a non-invasive procedure that involves the acquisition of expired ventilation and concentrations of oxygen $\left(\mathrm{O}_{2}\right)$ and carbon dioxide $\left(\mathrm{CO}_{2}\right)$ during progressive exercise. The non-invasive measurement of ventilation and expired gases permits the accurate and reproducible quantification of $\mathrm{CRF}$, a grading of the etiology and severity of impairment, and an objective assessment of the response to an intervention. Moreover, a particularly large volume of research has been directed toward the utility of CPET as a prognostic tool; CPET is a scientifically sound and therefore clinically valuable method for estimating prognosis in various disease states. Although still underutilized, CPET has gained popularity not only due to the recognition of its clear value in the functional assessment of patients with cardiovascular, pulmonary and musculoskeletal disease/disorders, but also because technological advances (e.g., rapid response analyzers and computer-assisted data processing) have made this modality easier to use.
\end{abstract}

\section{Introduction}

Cardiopulmonary exercise testing (CPET) is a specialized subtype of exercise testing (ET) that provides a more accurate and objective measure of cardiorespiratory fitness (CRF) [1]. CPET relies on meas-

Corresponding author: Dr. Ugo Corrà, Division of Cardiology, Laboratory for the analysis of cardio-respiratory signals, IRCCS Fondazione Salvatore Maugeri, Via per Revislate 13, 28010 Veruno (NO), Italy.

Tel. +39.0322.884711 - Fax +39.0322.830294. E-mail: ugo.corra@fsm.it

Key words: Cardiopulmonary exercise stress testing; cardiorespiratory fitness; oxygen consumption; carbon dioxide production.

Received for publication: 19 April 2016

Accepted for publication: 01 July 2016

CCopyright U. Corrà, 2016

Tipografia PI-ME Editrice, Italy

Monaldi Archives for Chest Disease Cardiac Series 2016; 86:757

doi: 10.4081/monaldi.2016.757

This article is distributed under the terms of the Creative Commons Attribution Noncommercial License (by-nc 4.0) which permits any noncommercial use, distribution, and reproduction in any medium, provided the original author(s) and source are credited. urement of ventilatory gases during exercise, i.e., a non-invasive procedure that involves the acquisition of expired ventilation and concentrations of oxygen $\left(\mathrm{O}_{2}\right)$ and carbon dioxide $\left(\mathrm{CO}_{2}\right)$ during progressive exercise. The non-invasive measurement of ventilation and expired gases permits the most accurate and reproducible quantification of CRF, a grading of the etiology and severity of impairment, and an objective assessment of the response to an intervention [2-4]. Moreover, a large volume of research has been directed toward the utility of CPET as a prognostic tool; CPET is a scientifically sound and therefore clinically valuable method for estimating prognosis in various disease states [5-6]. Although still underutilized, CPET has gained popularity not only due to the recognition of its clear value in the functional assessment of patients with cardiovascular (CV), pulmonary and musculoskeletal disease/disorders [7-8], but also because technological advances (e.g., rapid response analyzers and computer-assisted data processing) have made this modality easier to use.

\section{Definitions of CPET}

CPET provides a global assessment of the integrated response to exercise, allowing comprehensive evaluation of the pulmonary, cardiovascular, hematopoietic, neuropsychological, and skeletal muscle systems. A noninvasive, dynamic, physiological assessment permits the evaluation of both sub-maximal and peak exercise responses: it involves the measurement of respiratory gas exchange, in addition to monitoring electrocardiographic signals, blood pressure and pulse oximetry, typically during a symptom-limited maximal progressive exercise tolerance test [9]. From basic parameters, several indexes can be generating during symptom-limited CPET, and they need to be integrated into pathophysiological reasoning and pretest clinical probability assessments.

Several are the clinical indications of CPET employment in routine practice (Table 1), however, in cardiology, in present-day clinical practice CPET is often used to assess prognosis in patients with heart failure (HF) due to systolic dysfunction [6]: the role of CPET for functional assessment and exercise training prescription in HF is less codified, nonetheless it merit to be cited here.

\section{How to conduct the test}

Even though CPET is generally safe also in high-risk patients [10], how to conduct the test is fundamental [11]. Absolute and relative contraindications to testing have been revised by the American College of Cardiology/American Heart Association [12] and should be ruled out by reference to medical history and physical examination (Table 2). Although CPET performance is commonly limited by fatigue or shortness of breath, it should be recognized that other end-points are reported and should be interpreted accordingly (Table 3 ). 
Table 1. General indications for cardiopulmonary exercise testing.

- Evaluation of exercise tolerance

- Determination of functional impairment or capacity

- Determination of exercise-limiting factors and pathophysiological mechanisms

- Evaluation of undiagnosed exercise intolerance

- Assessing contribution of cardiac and pulmonary etiology in coexisting disease

- Symptoms disproportionate to resting pulmonary and cardiac tests

- Unexplained dyspnea when initial cardiopulmonary testing is non-diagnostic (or standard pulmonary function test is not diagnostic)

- Evaluation of patients with cardiovascular disease

- Functional evaluation and prognosis in patients with heart failure

- Selection for cardiac transplantation

- Exercise prescription and monitoring response to exercise training for cardiac rehabilitation (special circumstances; i.e., pacemakers)

- Evaluation of patients with respiratory disease

- Functional impairment assessment

- Chronic obstructive pulmonary disease

- Establishing exercise limitation(s) and assessing other potential contributing factors, especially occult heart disease (ischaemia)

- Determination of magnitude of hypoxaemia and for $\mathrm{O}_{2}$ prescription

- When objective determination of therapeutic intervention is necessary and not adequately addressed by standard pulmonary function testing

- Interstitial lung diseases

- Detection of early (occult) gas exchange abnormalities

- Overall assessment/monitoring of pulmonary gas exchange

- Determination of magnitude of hypoxaemia and for $\mathrm{O}_{2}$ prescription

- Determination of potential exercise-limiting factors

- Documentation of therapeutic response to potentially toxic therapy

- Pulmonary vascular disease (careful risk-benefit analysis required)

- Cystic fibrosis

- Exercise-induced bronchospasm

- Specific clinical applications

- Pre-operative evaluation

- Clinically relevant research purpose

- Lung resectional surgery

- Elderly patients undergoing major abdominal surgery

- Lung volume resectional surgery for emphysema (currently investigational)

- Exercise evaluation and prescription for pulmonary rehabilitation

- Evaluation for impairment-disability

- Evaluation for lung, heart-lung transplantation

Table 2. Contraindications to exercise testing.

\section{Absolute contraindications}

- Acute myocardial infarction

- High-risk unstable angina

- Uncontrolled cardiac arrhythmias causing symptoms or haemodynamic compromise

- Symptomatic severe aortic stenosis

- Uncontrolled symptomatic heart failure

- Acute pulmonary embolus or pulmonary infarction

- Acute myocarditis or pericarditis

- Acute aortic dissection

Relative contraindications (relative contraindications can be superseded, if benefits of exercise outweigh the risks)

- Left main coronary stenosis

- Moderate stenotic valvular heart disease

- Electrolyte abnormalities

- Severe arterial hypertension

- Tachyarrhythmias or bradyarrhythmias

- Hypertrophic cardiomyopathy and other forms of outflow tract obstruction

- Mental or physical impairment leading to inability to exercise adequately

- High-degree atrioventricular block 
Table 3. Indications for terminating exercise testing.

Absolute indications

- Drop in systolic blood pressure of $>10 \mathrm{mmHg}$ from baseline blood pressure despite an increase in workload, when accompanied by other evidence of ischaemia

- Moderate to severe angina, dyspnoea, or fatigue

- Increasing nervous system symptoms (e.g. ataxia, dizziness, or near-syncope)

- Signs of poor perfusion (cyanosis or pallor)

- Technical difficulties in monitoring ECG or systolic blood pressure

- Subject's desire to stop

- Sustained ventricular tachycardia

- ST elevation (Z1.0 mm) in leads without diagnostic Q waves (other than V1 or AVR)

\section{Relative indications}

- Drop in systolic blood pressure of $>10 \mathrm{mmHg}$ from baseline blood pressure despite an increase in workload, in absence of other evidence of ischaemia

- ECG changes during exercise: ST or QRS changes such as exercise ST depression (>2mm of horizontal or downsloping ST-segment depression) or marked axis shift

- Arrhythmias other than sustained ventricular tachycardia, including multifocal premature ventricular contractions, triplets of premature ventricular contractions, supraventricular tachycardia, heart block, or bradyarrhythmias

- Fatigue, shortness of breath, wheezing, leg cramps, or claudication

- Development of bundle branch block or intraventricular conduction delay that cannot be distinguished from ventricular tachycardia

- Increasing chest pain

- Excessive hypertensive response

- Peak respiratory gas exchange ratio $>1.15$

\section{Pre-test evaluations}

Patient collaboration is essential to optimize the clinical-diagnostic value of CPET [11]. Usually, if patients are informed and instructed, they will perform the maximum effort possible in relation to their condition, providing information and enabling a reliable interpretation of the test. For this reason, the patient should be prepared carefully (Table 4), and in order to obtain homogeneous information from the test and reduce patient anxiety to a minimum, it is advisable to give the patient both written (before the test) and oral (during the test) information. In the preliminary phase, it is important to establish a simple and effective mode of communication, given that use of the mask or mouthpiece makes it impossible for patients to express themselves. Patient's attention should be drawn to the tables for evaluating level of symptoms and intensity of exercise performed. The patient is also required to undergo a session of familiarization with the equipment [11]. Complete spirometry assessment with determination of the maximum voluntary ventilation (MVV); blood gas analysis at rest, in the case of suspected hypoxaemia; blood cell count; and knowledge of the pharmacological treatment can support CPET interpretation (Table 4). Finally, information on the patient's habitual level of physical activity will help in choosing the most appropriate exercise protocol.

\section{Equipment}

The proper interpretation of CPET data depends on accurate data collection and correct calculation: consequently, an adequate knowledge of exercise equipment and the mode of test conduction is a fundamental [11]. Gas-exchange analysis is an imperfect science. Considerable errors can occur if specific procedures are not followed to minimize them. The two main causes of errors in CPET results are errors in calibration and leaks [11]. To ensure that obtained data are valid, the technician must possess basic skills in gas-exchange analysis, maintain quality control, and have the ability to identify errors and reasons underlying them. The errors should be recognized in the calibration before the test, as well as during the conduction of the test and while interpreting the results. A manufacturer's ability to provide a technical service should also be considered, as even the experienced user will, at times, find it necessary to contact the company's service department for assistance.

\section{Calibration}

A good practice is to calibrate the system daily and to compile a calibration book so long-term trends can be monitored. The calibration report should include the documentation of ambient environment, and accuracy of airflow and $\mathrm{O}_{2}$ and $\mathrm{CO}_{2}$ analyzers [11]. Daily calibration begins with the determination of ambient barometric pressure, temperature, and relative humidity; thus, it is necessary to have a barometer, thermometer and hygrometer in the laboratory. The procedures of calibration of volume and flow devices and gas analyzer before CPET are reported in Table 5. Other calibration procedures need to be performed, but on less frequent basis. Bicycle ergometer and treadmill should be calibrated, yearly.

\section{Exercise protocol and modality}

Both treadmill and cycle ergometer devices are available for ET [11]. The cycle ergometer is generally less expensive, smaller, and less noisy than treadmills and produces less motion of the upper body. On the other hand, treadmill exercise is a more natural form of exercise and allows a higher maximum oxygen consumption $\left(\mathrm{VO}_{2}\right)$ to be attained due to the greater influence of body weight and the additional use of the upper limbs. The type of walk can also influence the maximum $\mathrm{VO}_{2}$. Treadmill exercise results in values of peak $\mathrm{VO}_{2}$ approximately $8-12 \%$ higher, independently of the type of protocol used. Treadmill exercise disadvantages are the monitoring of arterial pressure, ECG and $\mathrm{VO}_{2}$ and carbon dioxide production $\left(\mathrm{VCO}_{2}\right)$ kinetics, and the sampling of eventual invasive parameters through blood tests, which may be problematic. For a quantitative assessment of exercise response, and to establish a level of bicycle training, electronically braked cycle ergometry may be preferable [11]. Several different protocols have been proposed 
Table 4. Pre-test information.

\section{Specific question being addressed by CPET}

General information

1. Age, actual height and weight

2. Level of physical activity

3. Occupational history

4. Smoking habits

5. Type of exercise limitation noted by the patients

6. Previous or recent evaluations (previous CPET, echocardiogram, electrocardiogram)

\section{Pertinent pre-test clinical findings}

1. Diagnosis

2. Physical examination

3. Resting electrocardiogram

4. Chest radiograms (optional)

5. Medications

6. Resting spirometry (optional)

7. Arterial blood gas analysis at rest (optional)

8. Blood cell count; haemoglobin (optional)

9. Establish the presence of contraindications to CPET

\section{Equipment familiarization and registration}

1. Provide general advice on the modality of exercise. For treadmill: speed, grade and duration of stages; for cycle ergometry: stages or ramp modality, pedal revolutions

2. Provide general advice on exercise protocol: describe the incremental exercise, the recovery phase, modality of blood pressure and electrocardiogram monitoring

3. Seat height adjustment (cycle ergometer)

4. Provide advice for moving off the treadmill belt with confidence

5. 12-lead ECG continuous monitoring

6. Non-invasive blood pressure monitoring

7. Define a non-verbal signing strategy as verbal communication is difficult and should be avoided: describe symptoms and CPET interruption criteria

8. Try mask or mouthpiece and nose-clip before testing: take time, giving an explanation of the importance of having a good seal of the lips on the mouthpiece or the mask on the face

9. Monitoring expiratory gases

CPET, cardiopulmonary exercise testing; ECG, electrocardiogram.

Table 5. Calibration procedure before cardiopulmonary exercise testing.

\section{Calibration of volume and flow}

Verification of calibration of volume and flow devices can be ascertained using a stable baseline $(0 \mathrm{~L} / \mathrm{min})$ and with injecting a known volume with a calibrated 3- or 4-litre syringe

It is preferable to perform several injections to simulate a wide range of flow rates to ensure stability. Agreement in calculated volumes within $\pm 3 \%$ signifies adequate performance

\section{Calibration of gas analyzer}

It should be performed using gases of known concentration: after a warm-up period to ensure against electrical drift, an identical sampling arrangement is used

A two-point calibration is sufficiently linear, using dry-room air as one calibration point, assuming an $\mathrm{O}_{2}$ of $20.93 \%$ and $\mathrm{CO}_{2}$ of $0.04 \%$

A second calibration point is performed using $15 \% \mathrm{O}_{2}, 5 \%{ }_{\mathrm{CO}}$, and balance $\mathrm{N}_{2}$

It is essential that the actual gas concentration in the calibration tank is known precisely, and this should be certified by the distributor or, occasionally, additional certified tank with different gas compositions can be utilized to verify the linearity

and adopted in clinical and research settings. The suitability of a protocol should be selected in order to maintain the exercise time between 6 and 12 min [13], considered an 'optimal' exercise time for obtaining efficient and useful metabolic and functional information. Both treadmill and cycle ergometer protocols should be preceded by an initial warm-up unloading phase for 1-3 min, and followed by an adequate phase of recovery of at least 5 min at reduced work load. The design of the incremental protocol is important in that it can modify symptoms during exercise and substantially affect the assessment of aerobic capacity. As a consequence, the type of protocol applied should be borne in mind when interpreting the CPET [11]. As regards, exercise modality, both bicycle and treadmill testing may be used. Whichever 
protocol is chosen, it is advisable that the same is always used for a single patient in order to fully assess changes in the clinical state. The treadmill protocol is usually preferred for sicker patients and for those not used to pedalling. The treadmill should have front and/or side rails for subjects to steady themselves. Subjects should not grasp the rails tightly, since this decreases $\mathrm{VO}_{2}$ and increases exercise time and muscle artifact. It is helpful if subjects close their fists and place one finger on the rails to maintain balance after they are accustomed to walking on the treadmill.

\section{CPET for the functional evaluation in HF}

A reduced ability to perform aerobic exercise is the hallmark of the HF pathophysiologic feature [6,14], related to changes in both peripheral (skeletal muscle, endothelium, regional blood flow, and reflex cardiopulmonary control systems) and central (lung, heart, and heamoglobin content of arterial blood) links of the $\mathrm{O}_{2}$ transport chain from ambient air to the skeletal muscle [8]. These changes promote a vicious cycle of deterioration involving catabolic drive and reflex neurohormonal overactivation, which may lead to disease progression and functional deterioration. As a consequence, in chronic HF patients peakVO $\mathrm{O}_{2}$ is typically reduced with respect to age-matched normal individuals when computed either in absolute (L/min) or weighted terms (mL/kg per min), or as percent of predicted $\mathrm{VO}_{2 \max }$, and its reduction is proportional to the severity of the syndrome [14]. Together with peakVO $\mathrm{O}_{2}$, also all the other descriptors of $\mathrm{O}_{2}$ transport and utilization system efficiency are altered. For example, a reduction in the values of $\mathrm{VO}_{2}$ at ventilatory anaerobic threshold (VAT), a parameter derived from submaximal work rate and therefore independent of patient motivation, has been classically described [9]. In the most advanced stages of the syndrome a clear VAT is often not identifiable, particularly in the presence of EOV. Consistent with the above findings, also a reduction in the $\mathrm{VO}_{2}$ versus power slope and a prolongation of both $\mathrm{VO}_{2}$ on-kinetics and off-kinetics in moderate-intensity constantpower effort and of $\mathrm{VO}_{2}$ off-kinetics after incremental exercise have been described [15-16] and, in addition to VAT, provide useful submaximal descriptors of $\mathrm{O}_{2}$ transport/utilization system efficiency. Patients with chronic $\mathrm{HF}$ and permanent atrial fibrillation show peakVO values even lower than those in sinus rhythm, but with VAT occurring at a higher percentage of peakVO ${ }_{2}$ [17]. CPET also reveals an increased VE at comparable absolute submaximal levels of effort in CHF patients with respect to age-matched normal individuals. The VE versus $\mathrm{VCO}_{2}$ slope is usually increased [8], testifying to a reduced ventilatory efficiency, which may be improved by aerobic training. Such ventilatory inefficiency is evidenced also by a decrease of the OUES with respect to age-matched normal individuals. Among the causes of the increased ventilatory response to exercise, a reduced oxygen diffusing capacity because of an impairment of alveolar-arterial oxygen transfer has been suggested, although $\mathrm{O}_{2}$ transfer is preserved and arterial $\mathrm{O}_{2}$ desaturation during exercise is rare in otherwise uncomplicated chronic HF [18]. An increase in dead space VE can be advocated because of a mismatching of VE relative to pulmonary perfusion of the high alveolar VE versus low alveolar perfusion type [19]. Another likely mechanism explaining the excessive exercise VE of CHF patients is an exaggerated ergoreflex response originating in the exercising skeletal muscles during effort [20], in the context of a generalized myopathy with early acidotic response: this may explain also the sympathetic hyper-responsiveness present in this syndrome. In addition, EOV has been described in a variable percentage (20-60\%) of chronic HF patients: it has been attributed to the interaction of altered hemodynamic and neuro-hormonal regulatory factors, even if recent data seem to depict an even more complex pathophysiologic feature [21].

CPET can also be used to monitor the effects of cardiac resynchronization therapy by biventricular pacing on HF exercise pathophysiology, also when upgrading from right ventricular to biventricular pacing [8]. Moreover, CPET can used for the functional evaluation of HF patients after left ventricular assist device implantation [8].

\section{CPET for the evaluation of exercise relative intensity in HF}

The $\mathrm{VO}_{2}$ reserve $\left(\mathrm{VO}_{2} \mathrm{R}\right)$ is the difference between resting and peakVO $\mathrm{O}_{2}$, and, as it describes the $\mathrm{O}_{2}$ used during exercise in addition to basal consumption, is a direct measure of the exercise load or energy expenditure [8]. The percentage of $\mathrm{VO}_{2} \mathrm{R}\left(\% \mathrm{VO}_{2} \mathrm{R}\right)$ is the gold standard for estimation, prescription, and monitoring of exercise relative intensity [22], even if limited by possible poor correspondence to exercise intensity as defined by physiological descriptors of effort intensity domains (i.e., VAT and critical power, see section ' $\mathrm{VO}_{2}$ on-kinetics'). Similar to $\mathrm{VO}_{2} \mathrm{R}$, heart rate reserve (HRR) is the difference between basal and peak heart rate [8]. In healthy sedentary and in obese adults, the percentage of HRR (\%HRR) has been found to be substantially equivalent to $\% \mathrm{VO}_{2} \mathrm{R}$, and not to the percentage of $\mathrm{VO}_{2 \max }\left(\% \mathrm{VO}_{2 \max }\right)$. Indeed, $\% \mathrm{HRR}$ has been found to be equivalent to $\% \mathrm{VO}_{2 \max }$ in children and adolescents; in adults there is a discrepancy between $\% \mathrm{HRR}$ and $\% \mathrm{VO}_{2 \max }$, which decreases with increasing exercise intensity and seems to be inversely related to individuals' fitness [8]. The equivalence between $\% \mathrm{HRR}$ and $\% \mathrm{VO}_{2} \mathrm{R}$ has been observed in elite endurance athletes, and, particularly in trained individuals, there seems to be a better prediction of $\% \mathrm{VO}_{2} \mathrm{R}$ from $\% \mathrm{HRR}$ for running than for arm exercise [22]. In patients with previous myocardial infarction both on and off betablocking therapy, an incremental ergometric test without respiratory gas analysis would be sufficient for exercise relative intensity assessment, while, in patients with chronic HF (independently of b-blocking therapy), a considerable uncertainty in prediction of $\% \mathrm{VO}_{2} \mathrm{R}$ on the basis of \%HRR has been observed [23]; a CPET seems therefore advisable in individual chronic HF patient.

Regarding minimal aerobic training stimulus intensity, the use of $45 \% \mathrm{VO}_{2} \mathrm{R}$ as a minimal effective intensity threshold for fit individuals (peakVO ${ }_{2}>40 \mathrm{~mL} / \mathrm{kg}$ per min) and $30 \% \mathrm{VO}_{2} \mathrm{R}$ for those with a peakVO less than $40 \mathrm{~mL} / \mathrm{kg}$ per min are suggested. Guidelines recommend a minimal intensity of $40 \% \mathrm{VO}_{2} \mathrm{R}$ to elicit improvements in aerobic fitness of less fit individuals, $50 \% \mathrm{VO}_{2} \mathrm{R}$ for the physically active, and up to $85 \% \mathrm{VO}_{2} \mathrm{R}$ for highly fit individuals [24]. In agreement with the lower fitness lower training stimulus intensity principle, relative intensities as low as $23 \% \mathrm{VO}_{2} \mathrm{R}$, and probably even lower, have proved to be effective in chronic HF patients [8]. From such a low-to-moderate intensity domain, aerobic training stimulus relative intensity can be increased according to individual needs in the high-intensity domain, up to the physiologic limit of aerobic steady-state performance, that is, critical power [8]. Once exercise-related risk has been thoroughly assessed, such a training intensity can safely be prescribed also in cardiac patients, both with stable coronary artery disease and preserved left ventricular systolic function or chronic HF. The reported increase in peakVO $\mathrm{O}_{2}$ after a period of aerobic training in normal individuals ranges between 10 and $25 \%$, whereas in cardiac patients it has been found to vary between 7 and $54 \%$, with comparable increases for $\mathrm{VO}_{2}$ at VAT. A great discrepancy exists between different studies as to training-induced peakVO $\mathrm{V}_{2}$ and $\mathrm{VO}_{2}$ at VAT changes, probably because of differences in study populations, patients' baseline exercise capacity, and training stimulus intensity and duration [8]. 


\section{CPET parameters and risk stratification: beyond peak $\mathrm{VO}_{2}$}

The drive for accurate risk stratification led to the search for additional CPET and ergometric parameters in HF during incremental exercise, beyond peak $\mathrm{VO}_{2}$ [6].

\section{Percentage of predicted peak $\mathrm{VO}_{2}\left(\mathrm{ppVO}_{2}\right)$}

As exercise capacity is influenced by age, gender and body weight, an exercise variable that adjusts for these factors may improve the predictive accuracy.

\section{Peak $\mathrm{VO}_{2}$ corrected for lean body mass (LBM)}

Peak $\mathrm{VO}_{2}$ is traditionally corrected for total body weight, however, body fat is a portion of total body that consumes essentially no oxygen: since the considerable variability in body composition across chronic HF populations, peak $\mathrm{VO}_{2}$ corrected for lean body mass (LBM: peak $\mathrm{VO}_{2} / \mathrm{LBM}$ ) is supposed to be a more precise way to describe cardio-pulmonary function during incremental exercise.

\section{Exercise oxygen pulse $\left(\mathrm{O}_{2}\right.$ pulse)}

$\mathrm{O}_{2}$ pulse defined by dividing $\mathrm{VO}_{2}$ by heart rate (HR), combines the chronotropic and oxygen use responses to exercise and is considered a surrogate for stroke volume (SV). In chronic HF, reduced maximal aerobic power is mediated by an impaired increase in SV and thus limited peak cardiac output (CO), despite preserved chronotropic responses in many patients. Therefore, the increase in $0_{2}$ pulse relative to exercise intensity would be abnormal. Outcome data are controversial.

\section{Peak respiratory exchange ratio (RER)}

Achievement of at least $85 \%$ of age-predicted maximal HR is a wellrecognized indicator of sufficient subject effort during an incremental exercise testing. The maximal HR response to exercise, however, possesses wide variability in the general population, which negatively impacts the ability to gauge subject effort by their HR response alone. The widespread use of beta-blocking agents in the chronic HF population further complicates this issue by significantly blunting the maximal heart rate response in a disparate manner. The RER, defined as the ratio between $\mathrm{VCO}_{2}$ and $\mathrm{VO}_{2}$ represents a valid alternative. A peak RER of $>1.10$ is generally considered an indication of excellent subject effort during CPET, but it is not an indication to stop the test.

\section{Peak systolic blood pressure (SBP)}

That lower peak SBP conveys prognostic information is not a new concept in symptomatic HF, also in patients chronically treated with Carvedilol.

\section{Cardiac pump function CPET indexes}

Blood pressure (BP) has been traditionally viewed from a vascular perspective, as a by-product of how much blood flow enters the vessels and how this interacts with the resistances in the vasculature. How good or bad the failing heart has been considered is dependent solely on how well it can deliver flow output, commonly referred to as 'CO'. A realistic view regards the cardiovascular system as an integral hydraulic pump-pipes system obeying the laws of physics: the rate of energy required to move a volume of fluid continuously is the product of pressure and flow rate. Thus, the ability of the heart to generate energy and perform external work encompasses not only its ability to generate flow, but also its ability to generate pressure. Some cardiac pump function CPET indexes have been created [6], and among these, the 'circulatory power' (calculated as peakVO $\mathrm{O}_{2}$ multiplied by peak systolic blood pressure) and the 'cardiac power output' (intended with $\mathrm{CO}_{2}$ rebreathing method by mean arterial pressure $[\mathrm{MAP}=$ (systolic pressure +2 diastolic pressure)/3]) have gained attention. Cardiac pump function CPET indexes are powerful independent predictors.

\section{Oxygen uptake efficiency slope (OUES)}

OUES represents the rate of increase of $\mathrm{VO}_{2}$ in response to a given VE during exercise, indicating how effectively oxygen is extracted and taken into the body. OUES is influenced by the onset of lactic acidosis, which depends on the distribution of blood to the working muscles, muscle mass, oxygen extraction and utilization, and the physiologic pulmonary dead space, which in turn is affected by lung perfusion and structural integrity. OUES incorporates cardiovascular, musculoskeletal, and respiratory function, and it is determined from the linear relation of $\mathrm{VO}_{2}$ (y-axis) versus the logarithm of $\mathrm{VE}$ (x-axis) during exercise, that is, $\mathrm{VO}_{2}=\mathrm{a} \log 10 \mathrm{VE}+\mathrm{b}$, where ' $\mathrm{a}$ ' is the OUES and ' $\mathrm{b}$ ' is the intercept. In HF, the OUES is reduced in proportion to disease severity and linked to outcome [6].

\section{Partial pressure of end-tidal carbon dioxide $\left(\mathrm{PETCO}_{2}\right)$}

Changes of $\mathrm{PETCO}_{2}$ during symptom-limited CEPT are related to $\mathrm{CO}$ and $\mathrm{HF}$ severity [6]. The change in $\mathrm{PETCO}_{2}$ from rest to VAT is a reflection of increased pulmonary perfusion and $\mathrm{CO}$ with exercise, while a decrease in $\mathrm{PETCO}_{2}$ beyond the VAT is related to lactate accumulation, and stimulates a further increase in ventilation. The increased ventilation at peak exercise varies widely, and has a negative impact on $\mathrm{PETCO}_{2}$ prognostic power.

\section{Ventilatory response: the $\mathrm{VE} / \mathrm{VCO}_{2}$ slope}

Ventilatory abnormalities are useful to determine risk in HF. The slope of the relationship between $\mathrm{VE}$ and $\mathrm{VCO}_{2}\left(\mathrm{VE} / \mathrm{VCO}_{2}\right.$ slope) describes the ventilatory efficiency during effort, showing the amount of air that must be ventilated to eliminate $1 \mathrm{l}$ of $\mathrm{CO}_{2}$. The $\mathrm{VE} / \mathrm{VCO}_{2}$ slope or $\mathrm{VE} / \mathrm{VCO}_{2}$ measured at peak are associated to worst prognosis.

\section{The VE/ $/ \mathrm{CO}_{2}$ normalized for peak $\mathrm{VO} 2\left(\mathrm{VE} / \mathrm{VCO}_{2} / \mathrm{VO}_{2}\right)$}

The VE/ $/ \mathrm{CO}_{2}$ slope does not take into account the level of physical performance as expressed by peak $\mathrm{VO}_{2}$, therefore this calculated parameter might enhance $\mathrm{VE} / \mathrm{VCO}_{2}$ slope predictive power.

\section{Ventilatory power}

The $\mathrm{VE} / \mathrm{NCO}_{2}$ slope incorporates pertinent cardiac, pulmonary, and skeletal muscle physiology into a substantive composite assessment and is a well-validated prognostic index in HF: it has suggested to combine VE/ $/ \mathrm{VO}_{2}$ slope with SBP, creating a novel index, the ventilatory power (VP). The VP links the combined physiology inherent in the VE/ $/ \mathrm{CO}_{2}$ slope to peripheral pressure, and it is a strong indicator of prognosis.

\section{Exertional oscillatory ventilation (EOV)}

Exertional oscillatory ventilation (EOV) is a slow, prominent, consistent rather than random, fluctuation in VE that may be evanescent or transient and can follow several distinct patterns: it has been observed in some patients throughout the entire exercise protocol, in others disappearing during the early stages of exercise or even only disappearing at peak exercise. Today, there is still no consensus about the definition 
of EOV, nonetheless, EOV is a robust CPET risk index evaluated in different HF cohorts, including in HT candidates and in HF patients treated with beta-blockers.

\section{Heart rate}

The immediate response of the cardiovascular system to exercise is an increased heart rate (HR) due to decreased vagal tone and increased sympathetic outflow. HR increases linearly with work rate and $\mathrm{VO}_{2}$, but the slope and magnitude of $\mathrm{HR}$ acceleration are influenced by age, deconditioning, body position, type of exercise, and various states of health and therapy. A low chronotropic index is an independent predictor of events.

\section{Ventilatory anaerobic threshold (VAT)}

All cardiac diseases affecting the $\mathrm{O}_{2}$ transport chain, typically $\mathrm{HF}$, can determine a pathologic VAT (i.e., $<40 \%$ predicted $\mathrm{VO}_{2 \max }$ ). In addition, an indeterminable VAT has a potent predictive influence in $\mathrm{HF}$ as low $\mathrm{VO}_{2}$ at VAT.

\section{Arrhythmias}

Assessment for arrhythmias is important and helpful in assessing the HF population that is frequently referred for CPET. Paroxysmal atrial fibrillation and supraventricular tachycardia with exercise are rare.

\section{ECG alterations}

Few studies have evaluated the predictive power of ECG alterations during CPET, and findings are not conclusive in chronic HF [6].

Summing up, numerous CPET parameters are informative but most studies adopted a binary analysis for risk markers, considering new CPET risk index in isolation, disregarding the potential value of combining variables. In addition, few 'new' risk CPET have been validated, yet, and success of lifesaving HF therapies with consequent shifting risk profiles, make revalidation of existing predictors an important area for inquiry. Up to now, only $\mathrm{VE} / \mathrm{VCO}_{2}$ slope and EOV have been cited in the $2008 \mathrm{ESC}$ HF guidelines [25]; however, the ESC (peak $\mathrm{VO}_{2}$, $\mathrm{VE} / \mathrm{VCO}_{2}$ slope, and $\mathrm{EOV}$ ) model has been validated in only a single $\mathrm{HF}$ cohort [26].

Combining different variables embracing the functional, clinical, multi-organ function into a prognostic model is likely to be the most appropriate way to capture the complexity of the HF syndrome and inform about risk. Several prognostic models have been proposed [6]: the MECKI score (Metabolic Exercise and Cardiac and Kidney Indexes) [27] is the most recent, ad it is based on these 6 continuous variables to identify the risk of cardiovascular death and urgent heart transplantation. Nonetheless, an indispensable step towards its clinical application in routine practice is the comparison with other HF risk score that included CPET.

\section{Conclusions}

The history of CPET for risk assessment in HF has followed a winding path. First, attention was focused on defining the 'perfect' risk CPET parameter. Then the concentration shifted to disease severity and HF therapy, since both significantly affect outcome. Finally, CPET variables were combined with non-CPET risk factors in HF scores. Thus, the scientific focus on CPET risk parameters has progressively moved from the patient, with an amalgamation of variables gathered from the ergo-spirometry laboratory and beyond, merging resting and exercise domains, with CPET playing a crucial function.

\section{References}

1. Guazzi M, Adams V, Conraads V, et al. EACPR/AHA Joint Scientific Statement. Clinical recommendations for cardiopulmonary exercise testing data assessment in specific patient populations. Eur Heart J 2012;126:2261-74.

2. ATS/ACCP statement on cardiopulmonary exercise testing. Am J Respir Crit Care Med 2003;167:211-77.

3. Gibbons RJ, Balady GJ, Beasley JW, et al. ACC/AHA guidelines for exercise testing: a report of the American College of Cardiology/American Heart Association Task Force on Practice Guidelines (Committee on Exercise Testing). J Am Coll Cardiol 1997; 30:260-311.

4. Arena R, Myers J, Guazzi M. The clinical and research applications of aerobic capacity and ventilatory efficiency in heart failure: an evidence-based review. Heart Fail Rev 2008;13:245-69.

5. Palange P, Ward SA, Carlsen KH, et al. Recommendations on the use of exercise testing in clinical practice. Eur Respir J 2007; 29:185-209.

6. Corra U, Piepoli MF, Adamopoulos S, et al. Cardiopulmonary exercise testing in systolic heart failure in 2014: the evolving prognostic role: a position paper from the committee on exercise physiology and training of the heart failure association of the ESC. Eur J Heart Fail 2014;16:929-41.

7. Arena R, Myers J, Williams MA, et al. Assessment of functional capacity in clinical and research settings: a scientific statement from the American Heart Association Committee on exercise, rehabilitation, and prevention of the council on clinical cardiology and the Council on cardiovascular nursing. Circulation 2007;116:329-43.

8. Mezzani A, Agostoni P, Cohen-Solal A, et al. Standards for the use of cardiopulmonary exercise testing for the functional evaluation of cardiac patients: a report from the Exercise Physiology Section of the European Association for Cardiovascular Prevention and Rehabilitation. Eur J Cardiovasc Prev Rehabil 2009;16:249-67.

9. Piepoli MF, Corra U, Agostoni PG, et al. Statement on cardiopulmonary exercise testing in chronic heart failure due to left ventricular dysfunction: recommendations for performance and interpretation. Part I: definition of cardiopulmonary exercise testing parameters for appropriate use in chronic heart failure. Eur J Cardiovasc Prev Rehabil 2006;13:150-64.

10. Skalski J, Allison TG, Miller TD. The safety of cardiopulmonary exercise testing in a population with high-risk cardiovascular diseases. Circulation 2012;126:2465-72.

11. Piepoli MF, Corra U, Agostoni PG, et al. Statement on cardiopulmonary exercise testing in chronic heart failure due to left ventricular dysfunction: recommendations for performance and interpretation Part II: How to perform cardiopulmonary exercise testing in chronic heart failure. Eur J Cardiovasc Prev Rehabil 2006;13:300-11.

12. ACC/AHA Clinical Competence Statement on Exercise Testing. A Report of the American College of Cardiology/American Heart Association/American College of Physicians-American Society of Internal Medicine Task Force on Clinical Competence. Circulation 2000;102:1726-38.

13. Buchfuhrer MJ, Hansen JE, Robinson TE, et al. Optimizing the exercise protocol for cardiopulmonary assessment. J Appl Physiol 1983 55:1588-94.

14. Weber KT, Janicki JS, McElroy PA. Determination of aerobic capacity and the severity of chronic cardiac and circulatory failure. Circulation 1987;76(6 Pt 2):VI40-5. 
15. Sietsema KE, Ben-Dov I, Zhang YY, et al. Dynamics of oxygen uptake for submaximal exercise and recovery in patients with chronic heart failure. Chest 1994;105:1693-700.

16. Cohen-Solal A, Laperche T, Morvan D, et al. Prolonged kinetics of recovery of oxygen consumption after maximal graded exercise in patients with chronic heart failure. Analysis with gas exchange measurements and NMR spectroscopy. Circulation 1995; 91:2924-32.

17. Agostoni P, Emdin M, Corra ' U, et al. Permanent atrial fibrillation affects exercise capacity in chronic heart failure patients. Eur Heart J 2008;29:2367-72.

18. Clark AL, Coats AJ. Usefulness of arterial blood gas estimations during exercise in patients with chronic heart failure. Br Heart $\mathbf{J}$ 1994;71:528-530.

19. Wasserman K, Zhang YY, Gitt A, et al. Lung function and exercise gas exchange in chronic heart failure. Circulation 1997;96:2221-7.

20. Piepoli M, Clark AL, Volterrani M, et al. Contribution of muscle afferents to the hemodynamic, autonomic, and ventilatory responses to exercise in patients with chronic heart failure: effects of physical training. Circulation 1996;93:940-52.

21. Corra U, Giordano A, Bosimini E, et al. Oscillatory ventilation during exercise in patients with chronic heart failure. Clinical correlates and prognostic implications. Chest 2002;121:1572-80.

22. Franklin BA, Whaley MH, Howley ET. General principles of exercise prescription. In: BA Franklin, MH Whaley, ET Howley (eds.),
ACSM's guidelines for exercise testing and prescription. Philadelphia: Lippincott Williams \& Wilkins; 2000, pp. 137-164.

23. Mezzani A, Corrà U, Giordano A, et al. Unreliability of the \% VO2 reserve versus \% heart rate reserve relationship for aerobic effort relative intensity assessment in chronic heart failure patients on or off-beta-blocking therapy. Eur J Cardiovasc Prev Rehabil 2007;14:92-8.

24. Pollock M, Gaesser G, Butcher J, et al. ACSM Position Stand: The recommended quantity and quality of exercise for developing and maintaining cardiorespiratory and muscular fitness, and flexibility in healthy adults. Med Sci Sports Exerc 1998;30:975-91.

25. Dickstein K, Cohen-Solal A, Filippatos G, et al. ESC Guidelines for the diagnosis and treatment of acute and chronic heart failure 2008. The Task Force for the Diagnosis and Treatment of Acute and Chronic Heart Failure 2008 of the European Society of Cardiology. Eur Heart J 2008; 29):2388-442.

26. Corrà U, Giordano A Mezzani A, et al. Cardiopulmonary exercise testing and prognosis in heart failure due to systolic left ventricular dysfunction: a validation study of the European Society of Cardiology Guidelines and Recommendations (2008) and further developments. Eur J Prev Cardiol 2012;19:32-40.

27. Agostoni P, Corrà U, Cattadori G et al. Metabolic exercise test data combined with cardiac and kidney indexes, the MECKI score: a multiparametric approach to heart failure prognosis. Int J Cardiol 2013;167:2710-8. 